Pacific Journal of Mathematics

ON THE LOCAL SPECTRUM AND THE ADJOINT 


\title{
ON THE LOCAL SPECTRUM AND THE ADJOINT
}

\author{
JAMES K. FINCH
}

\begin{abstract}
Let $X$ be a Banach space, $X^{*}$ the dual space, and suppose that $T$ is a closed linear operator on $X$. Assume that the domain of $T$ is dense in $X$, so that the adjoint operator $T^{*}$ is a closed linear operator on $X^{*}$. The local spectrum $\sigma(T, x)$ is defined below. In this paper we investigate some of the relations between $\sigma(T, x)$ and $\sigma\left(T^{*}, x^{*}\right)$. In particular we show that if $\sigma(T, x)$ and $\sigma\left(T^{*}, x^{*}\right)$ are both empty, then $x^{*} x=0$.
\end{abstract}

The resolvent function of $T$ is $R_{T}(\lambda)=(\lambda I-T)^{-1}$; it is an operator valued function, and is defined and analytic for $\lambda$ not in $\sigma(T)$, the spectrum of $T$. Setting $f_{x}(\lambda)=R_{T}(\lambda) x$, then $f_{x}$ is analytic and satisfies $(\lambda I-T) f_{x}(\lambda)=x$ for all $\lambda$ not in $\sigma(T)$. However, it may be possible to find analytic solutions to $(\lambda I-T) f(\lambda)=x$ for some (or all) values of $\lambda$ that are in the spectrum of $T$. So we are led to define a local resolvent function of $T$ at $x$ as a vector valued analytic function $f$ which satisfies $(\lambda I-T) f(\lambda)=x$. It is easily shown that for $\lambda$ not in $\sigma(T)$, the only local resolvent is $f(\lambda)=R_{T}(\lambda) x$. But for $\lambda$ in $\sigma(T)$, there may be more than one local resolvent function. The local resolvent set is the union of the domains of all the local resolvent functions. The point at infinity is included if there is a local resolvent function which is defined and bounded for $|\lambda|>r$. The local spectrum $\sigma(T, x)$ is the complement of the local resolvent set. Clearly $\sigma(T, x)$ is a closed subset of the spectrum $\sigma(T)$; it may be equal to $\sigma(T)$, properly contained in it, or even empty.

The operator $T$ has the single valued extension property if there is at most one local resolvent function defined near any $\lambda$ in $C$; that is, whenever both $f$ and $g$ are local resolvent functions defined near $\lambda$, then $f=g$ there. In this case, there is a unique local resolvent with maximal domain. It can be shown that $T$ has the single valued extension property iff $\sigma(T, x)$ is not empty for any $x \neq 0$. (Note that $\sigma(T, 0)$ is always empty; the local resolvent $f(\lambda)=0$ is defined for all $\lambda$.

If both $T$ and $T^{*}$ have the single valued extension property, then $\sigma(T, x) \cap \sigma\left(T^{*}, x^{*}\right)=\varnothing$ implies $x^{*} x=0$ (see Lemma 1). This is not true if $\sigma(T, x)=\varnothing$ for some $x \neq 0$. For then $x^{*} x=0$ for all $x^{*}$, from which it would follow that $x=0$. Similarly, it is not true if $\sigma\left(T^{*}, x^{*}\right)$ is empty. But in Theorem 3 we show that if both $\sigma(T, x)$ and $\sigma\left(T^{*}, x^{*}\right)$ are empty, then $x^{*} x=0$.

Suppose that $T$ does not have the single valued extension 
property. Then for some open set $D \subseteq C$ there are distinct local resolvent functions for some $x$. Subtracting them, we find that there is a nonzero analytic function $f: D \rightarrow X$ which satisfies $(\lambda I-T) f(\lambda)=0$ for $\lambda$ in $D$. For a fixed $\lambda_{0}$ in $D, f$ has a series expansion $f(\lambda)=\sum_{n=0}^{\infty} x_{n}\left(\lambda-\lambda_{0}\right)^{n}$. It is not hard to show that $\left(\lambda_{0} I-T\right) x_{n+1}=-x_{n},\left(\lambda_{0} I-T\right) x_{0}=0$, and $\left(\lambda_{0} I-T\right)^{n+1} x_{n}=0$. Also, $\sigma\left(T, x_{n}\right)$ is empty for $n=0,1,2, \cdots$. To show this, we give two resolvent functions for $x_{n}$, whose domains together cover the extended complex plane. Set

$$
\begin{aligned}
g_{n}(\lambda) & =\sum_{k=0}^{n}(-1) k\left(\lambda_{0} I-T\right)^{k} x_{n} /\left(\lambda-\lambda_{0}\right)^{k+1} \\
& =\sum_{k=0}^{n} x_{n-k} /\left(\lambda-\lambda_{0}\right)^{k+1} \text { for } \lambda \neq \lambda_{0},
\end{aligned}
$$

and

$$
h_{n}(\lambda)=\sum_{k=0}^{\infty} x_{n+1+k}\left(\lambda-\lambda_{0}\right)^{k} \quad \text { for } \quad\left|\lambda-\lambda_{0}\right|<r .
$$

The series for $h$ converges in the same disk as the series for $f$. Straightforward calculation shows that $(\lambda I-T) g_{n}(\lambda)=x_{n}$ for $\lambda \neq \lambda_{0}$. To show that $(\lambda I-T) h_{n}(\lambda)=x_{n}$ for $\left|\lambda-\lambda_{0}\right|<r$, first consider the finite sum $S_{m}(\lambda)=\sum_{k=0}^{m-1} x_{n+1+k}\left(\lambda-\lambda_{0}\right)^{k}$. Then $(\lambda I-T) S_{m}(\lambda)=x_{n}$ $x_{n+m}\left(\lambda-\lambda_{0}\right)^{m}$, which converges to $x_{n}$ as $m \rightarrow \infty$. But $S_{m} \rightarrow h_{n}$ as $m \rightarrow \infty$. Since $T$ is a closed operator, we have $(\lambda I-T) h_{n}(\lambda)=x_{n}$.

From the above remarks we see that when an operator $T$ does not have the single valued extension property, it acts something like a left shift operator. For a specific example, consider an orthonormal basis $\left\{e_{n}\right\}$ for a separable Hilbert space $H$. Let $T$ be the left shift: $T e_{n+1}=e_{n}$ and $T e_{0}=0$. Then we have $(\lambda I-T) f(\lambda)=0$ on $|\lambda|<1$ for $f(\lambda)=\sum_{0}^{\infty} e_{n} \lambda^{n}$. And so $\sigma\left(T, e_{n}\right)$ is empty for all $n$. Actually $\sigma(T, x)$ is contained in the unit circle $\{\lambda:|\lambda|=1\}$ for all $x$ in $H$, although $\sigma(T)=\{\lambda:|\lambda| \leqq 1\}$. The function $g_{x}(\lambda)=-\sum \lambda^{n} S^{n+1} x$ is a local resolvent defined on $|\lambda|<1$, where $S$ is the right shift: $S e_{n}=$ $e_{n+1}$. Further, it is not hard to see that $\sigma\left(T^{*}, x^{*}\right)$ is the entire unit disk $\{\lambda:|\lambda| \leqq 1\}$ for all $x^{*}$ in $H^{*}$. In Theorem 2 below we show that this behavior is typical. This example was first given by Kakutani; see Dunford and Schwartz, page 1932. Kakutani's formulation is on the Hilbert space of functions analytic on the unit disk.

LEMma 1 (Bishop 1959). Suppose $f$ and $g$ are analytic functions with range in $X$ and $X^{*}$ respectively, and which satisfy $(\lambda I-T) f(\lambda)=x$ and $\left(\lambda I^{*}-T^{*}\right) g(\lambda)=x^{*}$. If the union of the domains of $f$ and $g$ is the entire complex plane, and if $f$ or $g$ is bounded in a neighborhood of infinity, then $x^{*} x=0$. 
The proof is an application of Liouville's Theorem to the function $h(\lambda)=x^{*} f(\lambda)$ for $\lambda$ in the domain of $f$, and $h(\lambda)=g(\lambda) x$ otherwise.

THEOREM 2. Let $T$ be a closed linear operator with domain dense in a Banach space $X$. Suppose that $T$ does not have the single valued extension property, so that there is an analytec function $f: D \subseteq C \rightarrow X$ with $(\lambda I-T) f(\lambda)=0$ for $\lambda$ in $D$. Assume that $D$ is a connected open set. Then for each $x^{*}$ in $X^{*}$, either

(i) $x^{*}$ is identically zero on the subspace spanned by $\{f(\lambda): \lambda$ in $D$, or

(ii) $\sigma\left(T^{*}, x^{*}\right)$ contains all of $D$.

Proof. Let $Y$ be the closed linear span of $\{f(\lambda): \lambda$ in $D\}$. For any $\lambda_{0}$ in $D$, expand $f$ in a Taylor series around $\lambda_{0}, f(\lambda)=\sum x_{n}\left(\lambda-\lambda_{0}\right)^{n}$. Each $x_{n}$ is in $Y$, since

$$
x_{n}=(2 \pi i)^{-1} \int_{\Gamma} f(\lambda)\left(\lambda-\lambda_{0}\right)^{-n-1} d \lambda,
$$

where $\Gamma$ is a circle around $\lambda_{0}$ contained in $D$. Moreover, $Y$ is actually spanned by $\left\{x_{n}\right\}$. For if $x^{*} x_{n}=0$ for all $n$, then $x^{*} f(\lambda)=0$ wherever the series converges. But $x^{*} f(\lambda)$ is analyic on the connected set $D$, so $x^{*} f(\lambda)=0$ for $\lambda$ in $D$. It follows that $x^{*} y=0$ for all $y$ in $Y$, and so $\left\{x_{n}\right\}$ spans $Y$.

Now suppose that $\lambda_{0}$ is in $D$, but is not in the local spectrum $\sigma\left(T^{*}, x^{*}\right)$. Then, by definition, there is an analytic function $h$ defined near $\lambda_{0}$ with $\left(\lambda I^{*}-T^{*}\right) h(\lambda)=x^{*}$. For each $n, g_{n}(\lambda)=\sum_{k=0}^{n}(-1)^{k}\left(\lambda_{0} I-\right.$ $T)^{k} x_{n} /\left(\lambda-\lambda_{0}\right)^{k+1}$ satisfies $(\lambda I-T) g_{n}(\lambda)=x_{n}$ for $\lambda \neq \lambda_{0}$. Then from Lemma $1, x^{*} x_{n}=0$ for all $n$. Hence $x^{*}$ is identically zero on $Y$.

Suppose that the local spectrums $\sigma^{*}(T, x)$ the $\sigma\left(T^{*}, x^{*}\right)$ are both empty. Can we conclude that $x^{*} x=0$ ? The answer is yes, but not directly from Theorem 2, for we do not know that $x$ is in a subspace $Y$ as described there. Instead, we show that $x$ is in the sum of a finite number of such subspaces. Then since $x^{*}$ is identically zero on each subspace, it is zero on the sum. And then $x^{*} x=0$.

Suppose that $\sigma(T, x)$ is empty. Then in particular the point at infinity is not in $\sigma(T, x)$; by definition this means that there is a bounded function $g(\lambda)$ defined on $D=\{\lambda:|\lambda|>r\}$ which satisfies $(\lambda I-T) g(\lambda)=x$. This function $g$ has a special form.

Lemma 3. The function $g(\lambda)$ described above has the series representation $g(\lambda)=\sum_{n=0}^{\infty} T^{n} x / \lambda^{n+1}$.

Proof. Since $g$ is bounded for $\lambda$ in $D$, it must have a series 
representation $\sum_{n=0}^{\infty} x_{n} / \lambda^{n}$. Then $g(\lambda) \rightarrow x_{0}$ as $\lambda \rightarrow \infty$, and so $g(\lambda) / \lambda \rightarrow 0$. But $T(g(\lambda) / \lambda)=T(g(\lambda)) / \lambda=(\lambda g(\lambda)-x) / \lambda \rightarrow x_{0} . \quad T$ is a closed operator, hence $x_{0}=T(0)=0$. Now, $\lambda g(\lambda) \rightarrow x_{1}$ as $\lambda \rightarrow \infty$, and so $T(g(\lambda))=$ $\lambda g(\lambda)-x$ converges to $x_{1}-x$. But $g(\lambda) \rightarrow 0$; thus $0=T(0)=x_{1}-x$, or $x_{1}=x$.

We show that $T x_{n}=x_{n+1}$ for $n \geqq 1$, and therefore that $x_{n}=T^{n} x$, using induction. Let

$$
\begin{aligned}
h(\lambda) & =x_{n}+x_{n+1} / \lambda+x_{n+2} / \lambda^{2}+\cdots \\
& =\lambda^{n} g(\lambda)-\lambda^{n-1} x_{1}-\cdots-\lambda x_{n-1} .
\end{aligned}
$$

Then

$$
\begin{aligned}
T h(\lambda) & =\lambda^{n}(\lambda g(\lambda)-x)-\lambda^{n-1} x_{2}-\cdots-\lambda x_{n} \\
& =\lambda^{n+1} g(\lambda)-\lambda^{n} x_{1}-\cdots-\lambda x_{n} \\
& =x_{n+1}+x_{n+2} / \lambda+\cdots .
\end{aligned}
$$

Thus as $\lambda \rightarrow \infty, h(\lambda) \rightarrow x_{n}$ and $T h(\lambda) \rightarrow x_{n+1}$. Since $T$ is closed, we have $T x_{n}=x_{n+1}$.

THEOREM 4. Suppose $T$ is a closed linear operator on a Banach space $X$ with domain dense in $X$. If $\sigma(T, x)$ and $\sigma\left(T^{*}, x^{*}\right)$ are both empty, then $x^{*} x=0$.

Proof. The idea is to show that if $\sigma(T, x)$ is empty, then $x$ is in a finite sum of subspaces spanned by $f_{k}(\lambda)$, where $(\lambda I-T) f_{k}(\lambda)=0$ for $\lambda$ in the domain of $f_{k}$. It then follows from Theorem 2 that $x^{*} x=0$.

If $\sigma(T, x)$ is empty, then for every complex number $z$, there is an analytic function $g_{z}(\lambda)$ which satisfies $(\lambda I-T) g_{z}(\lambda)=x$ and which is defined (at least) on some disk $D_{z}$ around $z$. In particular, there is such a function $g_{\infty}(\lambda)$ defined on $D_{\infty}=\left\{\lambda:|\lambda| \geqq r_{0}\right\}$. Take $B_{z}$ an open disk around $z$ contained in $D_{z}$; and $B_{\infty}$ a neighborhood of $\infty$ contained in $D_{\infty}$. The $B$ 's form an open cover of the extended complex plane, which is compact. Hence a finite number of them, $B_{\infty}$, $B_{1}, \cdots, B_{n}$ also form an open cover. Let $g_{0}, g_{1}, \cdots, g_{n}$ be the corresponding functions. Now the idea is that where the domains of $g_{i}$ and $g_{j}$ overlap, the function $f_{j k}=g_{j}-g_{k}$ satisfies $(\lambda I-T) f_{j k}(\lambda)=0$. The problem is to show that $x$ is in the sum of the subspaces generated by all the $f_{j k}$ 's.

We may assume that no $B_{j}$ is in the union of the previous $B$ 's; drop any that is. We may also assume that no $B_{j}$ is in the union of the closure of the others, by taking the $B_{j}$ slightly smaller if necessary. Now define closed sets by $C_{0}=\operatorname{cl}\left(B_{\infty}\right)$ and $C_{k}=\operatorname{cl}\left(B_{k}-\right.$ $\left.C_{0}-\cdots-C_{k-1}\right)$. We see that $B_{\infty} \cup B_{1} \cup \cdots \cup B_{k}$ is contained in $C_{0} \cup \cdots \cup C_{k}$ and that $C_{k}$ is disjoint from $B_{\infty}, B_{1}, \cdots, B_{k-1}$. Thus the 
family $C_{k}$ is a closed cover of the extended complex plane. The sets $C_{k}$ may have more than one component, but each component is bounded by a finite number of circular arcs. Let $\Gamma_{j}$ be the boundary of $C_{j}$. Given a point on $\Gamma_{j}$, any neighborhood of that point contains interior points of $C_{j}$ and interior points of some other $C_{k}$; by the construction, the point must then be on $\Gamma_{k}$. That is, every point on $\Gamma_{j}$ is on at least one other $\Gamma_{k}$. Also, a point where three or more of the $\Gamma_{k}$ meet must be a point where two or more of the original circular boundaries of the $B_{k}$ meet. Only a finite number of such points exist, since distinct circles intersect in at most two points.

Note that $2 \pi i x=\sum_{j} \int_{\Gamma_{j}} g_{j}(\lambda) d \lambda$, where the integral around $\Gamma_{0}$ is in the counterclockwise direction and the others in the clockwise direction. This is true since $\int_{\Gamma_{j}} g_{j}=0$ for $j=1, \cdots, n$, and $\int_{\Gamma_{0}} g_{0}=$ $2 \pi i x$ from Lemma 3. Now consider an $\operatorname{arc} \gamma$ on $\Gamma_{j}$ that extends from one intersection of three or more $\Gamma_{k}$ to another, without passing through a third (if there are not two such intersections on $\Gamma_{j}$, let $\gamma=\Gamma_{j}$ ). Each interior point of $\gamma$ is on exactly one other $\Gamma_{k}$; in fact, all interior points are on the same $\Gamma_{k}$ since otherwise an intersection of three $\Gamma_{k}$ would be an interior point of $\gamma$. The paths of integration of $\int_{\Gamma_{j}} g_{j}$ and $\int_{\Gamma_{k}} g_{k}$ both contain $\gamma$, and the integrals "go over" $\gamma$ in opposite directions. Thus these portions of those integrals may be combined to $\pm \int_{\gamma}\left(g_{j}-g_{k}\right)$. Doing this for each arc on each $\Gamma_{j}$, we see that the expression for $2 \pi i x$ becomes a linear combination of integrals of the form $\int_{r}\left(g_{j}-g_{k}\right)$. Since $(\lambda I-T)\left(g_{j}-g_{k}\right)=0$, setting $\hat{f}_{j k}=g_{j}-g_{k}$, we have $x$ in the sum of the spaces spanned by the $f_{j k}$.

THeORem 5. Suppose that $T$ is as above, and in addition the range of $T$ is closed. If for every $x \neq 0$ the local spectrum $\sigma(T, x)$ contains the disk $D=\{\lambda:|\lambda| \leqq r\}$, then $T^{*}$ does not have the single valued extension property. In particular, there are elements $x^{*} \neq 0$ in $X^{*}$ for which $\sigma\left(T^{*}, x^{*}\right)$ is empty.

Proof. First $T$ is one-one. For if $T x=0$, then $f(\lambda)=x / \lambda$ is a local resolvent function defined for all $\lambda \neq 0$. And then $\sigma(T, x) \subseteq\{0\}$, contrary to our assumption. However, 0 is in the spectrum of $T$. Thus $T$ cannot be onto. Since the range of $T$ is closed, $T^{*}$ is onto but not one-one. This is sufficient to show that $T^{*}$ does no have the single valued extension property. For take any $x_{0}^{*}$ with $x_{0}^{*}=1$ and $T^{*} x_{0}^{*}=0$, and form a sequence $x_{0}^{*}, x_{1}^{*}, x_{2}^{*}, \cdots$ with $T^{*} x_{n+1}^{*}=x_{n}^{*}$. 
Because $T^{*}$ is an open mapping, this can be done so that $\left\|x_{n+1}^{*}\right\|<$ $k\left\|x_{n}^{*}\right\|$. It is then fairly easy to show that $f^{*}(\lambda)=\sum x_{n}^{*} \lambda^{n}$ converges for $|\lambda|<1 / k$ and satisfies $\left(\lambda I^{*}-T^{*}\right) f^{*}(\lambda)=0$.

\section{REFERENCES}

1. Errett Bishop, A duality theory for arbitrary operators, Pacific J. Math., 9 (1959), 379-397.

2. Nelson Dunford and Jacob Schwartz, Linear Operators, New York: Interscience, 1957.

3. James Finch, The single valued extension property on a Banach space, Pacific J. Math., 58 (1975), 61-69.

4. F. H. Vasilescu, Residual properties for closed operators on Frèchet spaces, Illinois J. Math., 15 (1971), 377-386.

Received October 12, 1979.

University of San Francisco

SAN Francisco, CA 94117 


\section{PACIFIC JOURNAL OF MATHEMATICS}

\section{EDITORS}

DONALD BABBITT (Managing Editor)

University of California

Los Angeles, CA 90024

Hugo RossI

University of Utah

Salt Lake City, UT 84112

C. C. MOORE and ANDREW OGG

University of California

Berkeley, CA 94720
J. DugundjI

Department of Mathematics

University of Southern California

Los Angeles, CA 90007

R. FINN and J. MILGRAM

Stanford University

Stanford, CA 94305

\section{ASSOCIATE EDITORS}
R. ARENS
E. F. BECKENBACH
B. H. NeUmanN
F. WOLF
K. YosHIDA

\section{SUPPORTING INSTITUTIONS}

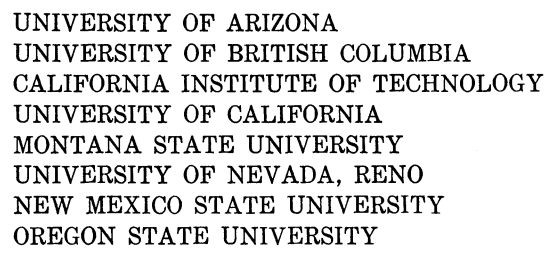

UNIVERSITY OF ARIZONA

UNIVERSITY OF BRITISH COLUMBIA CALIFORNIA INSTITUTE OF TECHNOLOGY

UNIVERSITY OF CALIFORNIA

MONTANA STATE UNIVERSITY

UNIVERSITY OF NEVADA, RENO

NEW MEXICO STATE UNIVERSITY OREGON STATE UNIVERSITY

\author{
UNIVERSITY OF OREGON \\ UNIVERSITY OF SOUTHERN CALIFORNIA \\ STANFORD UNIVERSITY \\ UNIVERSITY OF HAWAII \\ UNIVERSITY OF TOKYO \\ UNIVERSITY OF UTAH \\ WASHINGTON STATE UNIVERSITY \\ UNIVERSITY OF WASHINGTON
}

The Supporting Institutions listed above contribute to the cost of publication of this Journal, but they are not owners or publishers and have no responsibility for its content or policies.

Mathematical papers intended for publication in the Pacific Journal of Mathematics should be in typed form or offset-reproduced, (not dittoed), double spaced with large margins. Please do not use built up fractions in the text of the manuscript. However, you may use them in the displayed equations. Underline Greek letters in red, German in green, and script in blue. The first paragraph or two must be capable of being used separately as a synopsis of the entire paper. Please propose a heading for the odd numbered pages of less than 35 characters. Manuscripts, in triplicate, may be sent to any one of the editors. Please classify according to the scheme of Math. Reviews, Index to Vol. 39. Supply name and address of author to whom proofs should be sent. All other communications should be addressed to the managing editor, or Elaine Barth, University of California, Los Angeles, California, 90024.

50 reprints to each author are provided free for each article, only if page charges have been substantially paid. Additional copies may be obtained at cost in multiples of 50 .

The Pacific Journal of Mathematics is issued monthly as of January 1966. Regular subscription rate: $\$ 102.00$ a year (6 Vols., 12 issues). Special rate: $\$ 51.00$ a year to individual members of supporting institutions.

Subscriptions, orders for numbers issued in the last three calendar years, and changes of address shoud be sent to Pacific Journal of Mathematics, P.O. Box 969, Carmel Valley, CA 93924, U.S.A. Old back numbers obtainable from Kraus Per!odicals Co., Route 100, Millwood, NY 10546.

\section{PUBLISHED BY PACIFIC JOURNAL OF MATHEMATICS, A NON-PROFIT CORPORATION}

Printed at Kokusai Bunken Insatsusha (International Academic Printing Co., Ltd.). 8-8, 3-chome, Takadanobaba, Shinjuku-ku, Tokyo 160, Japan.

Copyright (C) 1981 by Pacific Jounal of Mathematics Manufactured and first issued in Japan 


\section{Pacific Journal of Mathematics}

Vol. 94, No. $2 \quad$ June, 1981

Thomas E. Armstrong and William David Sudderth, Nearly strategic

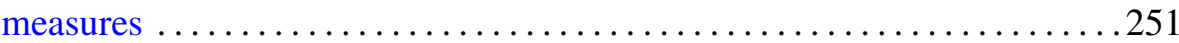

John J. Buoni, Artatrana Dash and Bhushan L. Wadhwa, Joint Browder

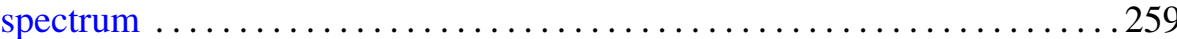

Jack Paul Diamond, Hypergeometric series with a $p$-adic variable . .......265

Raymond Frank Dickman, Jack Ray Porter and Leonard Rubin,

Completely regular absolutes and projective objects ............ 277

James Kenneth Finch, On the local spectrum and the adjoint ........... 297

Benno Fuchssteiner, An abstract disintegration theorem ............ 303

Leon Gerber, The volume cut off a simplex by a half-space $\ldots \ldots \ldots \ldots 311$

Irving Leonard Glicksberg, An application of Wermer's subharmonicity

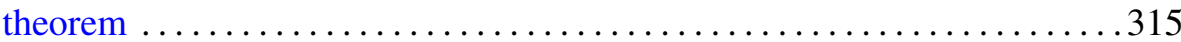

William Goldman, Two examples of affine manifolds ............... 327

Yukio Hirashita, On the Weierstrass points on open Riemann surfaces .....331

Darrell Conley Kent, A note on regular Cauchy spaces ............. 333

Abel Klein and Lawrence J. Landau, Periodic Gaussian

Osterwalder-Schrader positive processes and the two-sided Markov

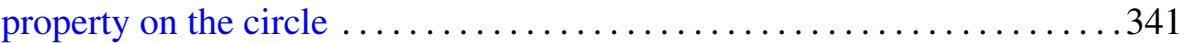

Brenda MacGibbon, $\mathscr{K}$-Borelian embeddings and images of Hausdorff

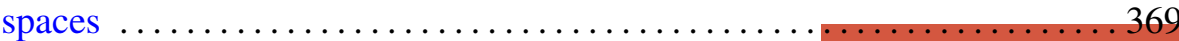

John R. Myers, Homology 3-spheres which admit no PL involutions . . . . . 379

Boon-Hua Ong, Invariant subspace lattices for a class of operators . . . . . 385

Chull Park, Representations of Gaussian processes by Wiener processes . . . 407

Lesley Millman Sibner and Robert Jules Sibner, A sub-elliptic estimate

for a class of invariantly defined elliptic systems $\ldots \ldots \ldots \ldots \ldots \ldots .417$

Justin R. Smith, Complements of codimension-two submanifolds. III.

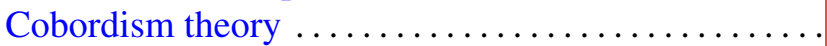

William Albert Roderick Weiss, Small Dowker spaces

David J. Winter, Cartan subalgebras of a Lie algebra and its ideals. II ... 